EPJ Web of Conferences 80, 00009 (2014)

DOI: $10.1051 /$ epjconf/20148000009

(C) Owned by the authors, published by EDP Sciences, 2014

\title{
Frequency dependence of anomalous transport in field theory and holography ${ }^{\star, \star \star}$
}

\author{
Eugenio Megías ${ }^{1, a}$ and Francisco Pena-Benitez $z^{2,3,4}$ \\ ${ }^{1}$ Grup de Física Teòrica and IFAE, Departament de Física, Universitat Autònoma de Barcelona, Bellaterra \\ 08193 Barcelona, Spain \\ ${ }^{2}$ Instituto de Física Teórica UAM/CSIC, Universidad Autónoma de Madrid, Cantoblanco 28049 Madrid, Spain \\ ${ }^{3}$ Departamento de Física Teórica, Universidad Autónoma de Madrid, Cantoblanco 28049 Madrid, Spain \\ ${ }^{4}$ Crete Center for Theoretical Physics, Department of Physics, University of Crete, 71003 Heraklion, Greece
}

\begin{abstract}
We study the frequency dependence of anomalous transport coefficients for a relativistic gas of free chiral fermions and for a strongly coupled conformal field theory with holographic dual. We perform the computation by using the Kubo formulae formalism, and compare with a hydrodynamic calculation of two point functions. Some implications for heavy ion physics are discussed.
\end{abstract}

\section{Introduction}

In the past few years it has been observed that quantum anomalies play a prominent role in the hydrodynamics of relativistic fluids. In presence of anomalies the currents are no longer conserved and this has important consequences in the constitutive relations for hydrodynamics. Two relevant effects appear at first order in the hydrodynamical expansion: the chiral magnetic effect (CME), which is responsible for the generation of an electric current parallel to a magnetic field, $\vec{J}=\sigma^{\mathscr{B}} \vec{B}[1]$, and the chiral vortical effect (CVE), in which the electric current in induced by a vortex, $\vec{J}=\sigma^{\mathscr{V}} \vec{\Omega}$ [2]. It is believed that these phenomena can produce observable effects in heavy ion physics [3], as well as in condensed matter systems [4]. While initially only pure gauge anomalies were considered to be relevant at first order, recent findings show that mixed gauge-gravitational anomalies contribute also in this case. This was first observed from Kubo formulae [5, 6], and later confirmed by other methods, including fluid/gravity [7, 8] and partition function formalism [9]. At second order a plethora of dissipative and non-dissipative conductivities including both anomalies have been studied [8, 10-13].

The CME and CVE require inhomogeneities in the system in order for a current being produced, and to this end a frequency-momentum dependence analysis is relevant. In this paper we will study this by using field theory and holographic methods, and finally extract some consequences for heavy ion physics.

\footnotetext{
*Talk given by E. Megías at the QCD@ Work: International Workshop on QCD, 16-19 June 2014, Giovinazzo, Bari, Italy.

${ }^{\star}$ Supported by Plan Nacional de Altas Energías (FPA2011-25948, FPA2012-32828), Spanish Consolider-Ingenio 2010 Programme CPAN (CSD2007-00042), Comunidad de Madrid HEP-HACOS S2009/ESP-1473, Spanish MINECO's Centro de Excelencia Severo Ochoa Program (SEV-2012-0234, SEV-2012-0249), the EU 7th Framework Programme (FP7-REGPOT2012-2013-1) no 316165, PIF-GA-2011-300984, the EU Programme "Thales" and "HERAKLEITOS II" ESF/NSRF 20072013, and the Greek Programme "Education and Lifelong Learning”. E.M. is supported by the Juan de la Cierva Program.

ae-mail: emegias@ifae.es
} 


\section{Transport coefficients from Kubo formulae}

Kubo formulae constitute an important tool for the computation of dissipative and non-dissipative conductivities. These are expressions which follow from linear response theory and involve retarded correlators of the currents and/or the energy-momentum tensor of the system. The Kubo formulae for the chiral magnetic $(\mathrm{CMC})$ and chiral vortical (CVC) conductivities write respectively $[1,6,14,15]$

$$
\sigma_{A}^{\mathscr{B}}(\omega, \vec{k})=-\sum_{i, j} \varepsilon_{i j n} \frac{i}{2 k_{n}}\left\langle J_{A}^{i} J_{e}^{j}\right\rangle, \quad \sigma_{A}^{\mathscr{V}}(\omega, \vec{k})=-\sum_{i, j} \varepsilon_{i j n} \frac{i}{2 k_{n}}\left\langle J_{A}^{i} J_{\varepsilon}^{j}\right\rangle, \quad J_{A}^{i} \in\left\{J_{e}^{i}, J_{5}^{i}, J_{\varepsilon}^{i}\right\},
$$

where $J_{e, 5}^{i}$ are charge currents and $J_{\varepsilon}^{i} \equiv T^{0 i}$ is the energy current. Unless otherwise indicated, in this manuscript we will be considering the symmetry group $U_{V}(1) \times U_{5}(1)$ so that there are vector and axial charges with chemical potentials $\mu_{ \pm}=\mu \pm \mu_{5}$. We will compute in this section the Kubo formulae of Eq. (1) at weak and strong coupling.

\subsection{Transport coefficients in the weakly coupled regime}

Let us consider a free field theory of chiral fermions. The charge and energy currents are, respectively,

$$
J_{e, 5}^{i}(x)=\Psi(x) \gamma^{i} Q_{e, 5} \Psi(x), \quad J_{\varepsilon}^{i}(x)=\frac{i}{4} \bar{\Psi}(x)\left(\gamma^{0} \overleftrightarrow{\partial^{i}}+\gamma^{i} \overleftrightarrow{\partial^{0}}\right) \Psi(x), \quad Q_{e, 5}=1, \gamma_{5}
$$

The problem of computing the conductivities reduces to the evaluation of the retarded correlators

$$
G_{A B} \equiv G_{A B}\left(x-x^{\prime}\right)=\frac{i}{2} \varepsilon_{i j n} \theta\left(t-t^{\prime}\right)\left(\left\langle\left[J_{A}^{i}(x), J_{B}^{j}\left(x^{\prime}\right)\right]\right\rangle+\delta_{A, \varepsilon} \delta_{B, \varepsilon} \Pi_{2}^{0 i 0 j}\left(x-x^{\prime}\right)\right), \quad A, B=e, 5, \varepsilon .
$$

The last term in the rhs is the seagull diagram contribution, and this comes from [16]

$$
\Pi_{2}^{\mu v \rho \sigma}=-4\left\langle\frac{\delta^{2} S_{2}}{\delta h_{\mu v} \delta h_{\rho \sigma}}\right\rangle
$$

where $S_{2}$ is the second order in an expansion of the action in powers of fluctuations of the metric $h_{\mu v}=g_{\mu v}-\eta_{\mu v}$. In the notation of Eq. (1), the CMC and CVC follow from the correlators $\sigma_{A}^{\mathscr{B}} \sim G_{A e}$ and $\sigma_{A}^{\mathscr{Y}} \sim G_{A \varepsilon}$. An explicit evaluation in the zero frequency-momentum limit gives $[1,5,15,17]$

$$
\sigma_{A,(0)}^{\mathscr{B}}=\frac{1}{2 \pi^{2}}\left\{\begin{array}{l}
\mu_{5} \\
\mu \\
\mu \mu_{5}
\end{array}, \quad \sigma_{A,(0)}^{\mathscr{V}}=\frac{1}{2 \pi^{2}}\left\{\begin{array}{l}
\mu \mu_{5} \\
\frac{\mu^{2}+\mu_{5}^{2}}{2}+\frac{\pi^{2} T^{2}}{6} \\
\mu_{5}\left(\frac{\mu^{2}+\mu_{5}^{2}}{3}+\frac{\pi^{2} T^{2}}{3}\right)
\end{array} \quad, \quad A=e, 5, \varepsilon .\right.\right.
$$

In the rest of the paper we will focus on the frequency and momentum dependence of these conductivities. In particular, in the limit $\vec{k} \rightarrow 0$ (and finite $\omega$ ) the imaginary part of the CMC writes [1, 17]

$$
\operatorname{Im}\left[\sigma_{A}^{\mathscr{B}}(\omega, 0)\right]=\left(2+\delta_{A, \varepsilon}\right) \frac{\pi}{3} \sigma_{A,(0)}^{\mathscr{B}} \omega \delta(\omega)+\frac{\omega|\omega|}{96 \pi} \sum_{s, t= \pm} t \zeta_{A}\left[\frac{d}{d x} n\left(x+t \mu_{s}\right)\right]_{x=|\omega| / 2}, A=e, 5, \varepsilon,
$$

where $n(x)=1 /\left(e^{\beta x}+1\right)$ is the Fermi-Dirac distribution function and $\zeta_{A}=s \delta_{A, e}+\delta_{A, 5}$. This formula in the low temperature limit develops resonances at $\omega= \pm 2 \mu_{s}$ which are related to particle-antiparticle pair production. Much more intriguing is the result for the CVC

$$
\sigma_{A}^{\mathscr{V}}(\omega, 0)=\sigma_{A,(0)}^{\mathscr{V}}\left(\delta_{\omega, 0}+i \pi \omega \delta(\omega)\right), \quad A=e, 5, \varepsilon,
$$

which leads to a vanishing value when $\omega \neq 0$ [17]. It is shown in Fig. 1 the behavior of the CMC and CVC at finite $\omega$ and $k$. The plot for the CVC shows $\sigma_{A}^{\mathscr{V}}(\omega, k) \approx 0$ when $k \ll \omega$, in agreement with Eq. (7). Some implications of this behavior in heavy ion collisions will be discussed in Sec. 4. 


\section{QCD@Work 2014}
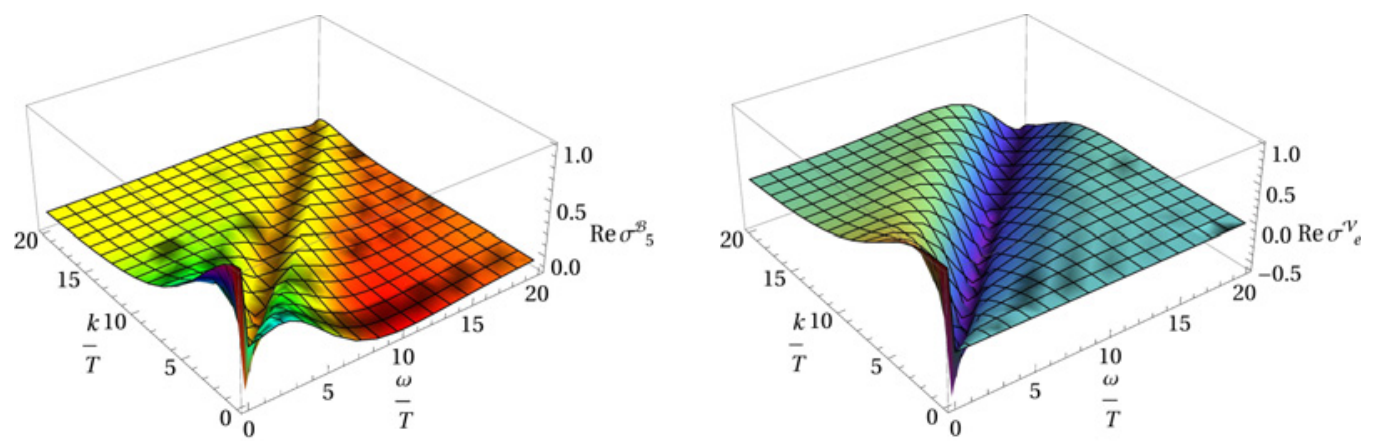

Figure 1. Frequency and momentum dependence of the real part of the axial magnetic conductivity $\sigma_{5}^{\mathscr{B}}(\omega, k)$ (left) and vector vortical conductivity $\sigma_{e}^{\mathscr{V}}(\omega, k)$ (right) from the weak coupling approach of Sec. 2.1. The conductivities are normalized to their zero frequency-momentum values. We consider $\mu=10 \mathrm{MeV}, \mu_{5}=1 \mathrm{MeV}$, and temperature above the QCD phase transition $\tau=440$, where $\tau=2 \pi T / \mu$.
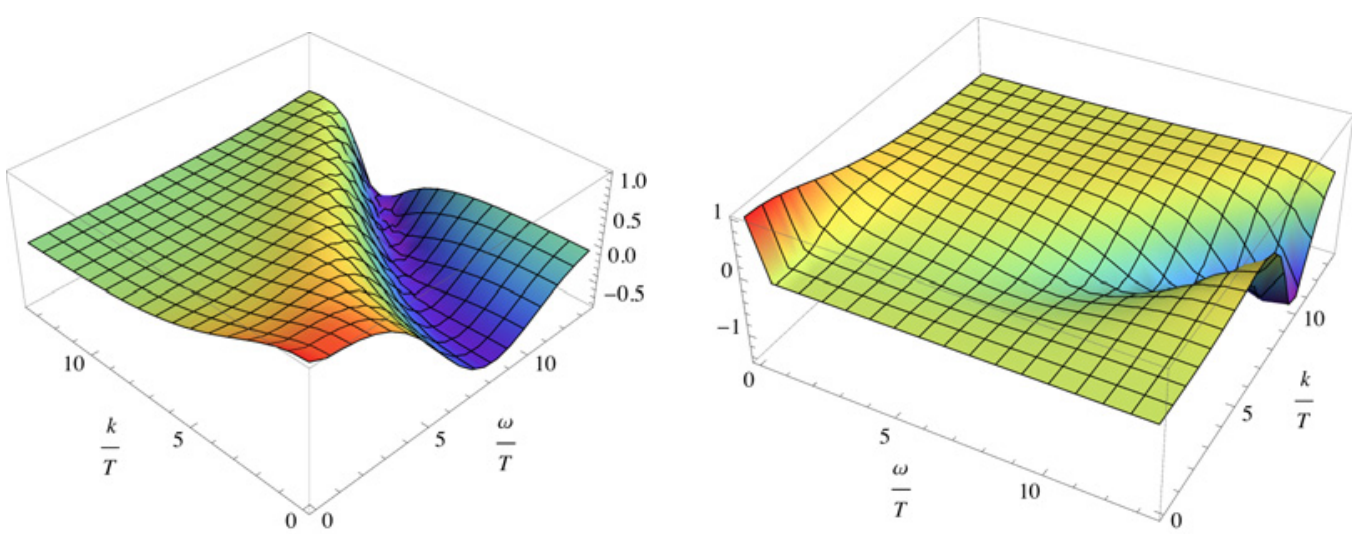

Figure 2. Frequency and momentum dependence of the real part of $\sigma_{5}^{\mathscr{B}}(\omega, k)$ (left) and $\sigma_{e}^{\mathscr{V}}(\omega, k)$ (right) from the strong coupling approach of Sec. 2.2. See Fig. 1 for further details.

\subsection{Transport coefficients in the strongly coupled regime}

To study the conductivities of a strongly coupled plasma we consider a Einstein-Maxwell model in 5-dim, supplemented with pure gauge and mixed gauge-gravitational Chern Simons terms ${ }^{1}[6,17]$

$$
S=\frac{1}{16 \pi G} \int d^{5} x \sqrt{-g}\left[R+2 \Lambda-\frac{1}{4} F_{M N}^{(5)} F^{(5) M N}+\varepsilon^{M N P Q R} A_{M}^{(5)}\left(\frac{\kappa}{3} F_{N P}^{(5)} F_{Q R}^{(5)}+\lambda R_{B N P}^{A} R^{B}{ }_{A Q R}\right)\right] .
$$

The explicit dependence on $A_{M}^{(5)}$ leads to the anomalous Ward identity in the case of a single $U(1)$ global current, that fixes the values of $\kappa$ and $\lambda$. This theory has a Reissner-Nordström black hole

\footnotetext{
${ }^{1} \mathrm{~A}$ computation with the symmetry group $U_{V}(1) \times U_{5}(1)$ demands the inclusion of vector and axial gauge fields, however for simplicity we write here the axial field only. In addition, some boundary terms should be added in Eq. (8) to make the action finite, but we neglect them for notational convenience. See [17] for complete details on the computation.
} 
solution

$$
d s^{2}=r^{2}\left(-f(r) d t^{2}+d \vec{x}^{2}\right)+\frac{1}{r^{2} f(r)} d r^{2}, \quad A_{(0)}^{(5)}=-\frac{\mu_{5} r_{h}^{2}}{r^{2}} d t
$$

where

$$
f(r)=1-\frac{m}{r^{4}}+\frac{q^{2}}{r^{6}}
$$

is the blackening factor, while $m$ and $q$ are the mass and charge of the black hole which are related to the temperature and chemical potential through $T=\frac{1}{2 \pi r_{h}^{3}}\left(2 m-3 q^{2} / r_{h}^{2}\right)$ and $q^{2}=\mu_{5}^{2} r_{h}^{4} / 3$. In these formulas $r_{h}$ is the outer horizon of the black hole.

To compute the two point retarded correlators we just need to use the AdS/CFT dictionary [18] and then plugging the formulas into the Kubo expressions Eq. (1). The zero frequency-momentum conductivities at strong coupling agree exactly with the weak coupling ones [6, 14, 17], cf. Eq. (5). To study the frequency dependence it is necessary to resort to numerics, and the results are plotted in Fig. 2. The behavior is qualitatively similar to the weakly coupled case. Note that Eq. (7) is also fulfilled at strong coupling, and this confirms that this is a general property which is independent of the interaction. As noted in [17], this behavior is in fact a natural consequence of Ward identities that include the energy-momentum tensor, as these imply $\omega \sigma_{A}^{\mathscr{V}}(\omega, 0)=0$.

\section{Two point functions in hydrodynamics}

The hydrodynamic approach is based on the assumption that the mean free path (time) of particles is much shorter than the characteristic size (time scale) of the system. In momentum space, this can be written as $k \ll 1 / \ell_{\mathrm{mfp}}$ and $\omega \ll 1 / \tau_{\mathrm{mft}}$. In this section we will compute the two point functions Eq. (1) predicted by hydrodynamics. The constitutive relations read ${ }^{2}$

$$
\begin{aligned}
\left\langle T^{\mu v}\right\rangle & =(\varepsilon+P) u^{\mu} u^{v}+P g^{\mu v}-\eta \sigma^{\mu v}+2 \sigma_{\varepsilon}^{\mathscr{B}} B^{(\mu} u^{v)}+2 \sigma_{\varepsilon}^{\mathscr{V}} \Omega^{(\mu} u^{v)} \\
\left\langle J^{\mu}\right\rangle & =\rho u^{\mu}+\sigma^{\mathscr{B}} B^{\mu}+\sigma^{\mathscr{V}} \Omega^{\mu}
\end{aligned}
$$

where $\varepsilon$ is the energy density, $P$ the pressure, $\rho$ the charge density and $u^{\mu}$ the fluid velocity. In addition we need the energy-momentum conservation $\nabla_{\mu} T^{\mu v}=F^{v \mu} J_{\mu}$. We consider fluctuations in the gauge field $\varepsilon a_{\mu}$ and in the metric $g_{\mu v}=\eta_{\mu v}+\varepsilon h_{\mu \nu}$. Then the retarded two point functions can be computed within the linear response theory by expanding the constitutive relations up to order $\mathscr{O}(\varepsilon)$, and using

$$
\left\langle T^{t i} T^{t j}\right\rangle=\frac{\partial}{\partial h_{t j}}\left\langle T^{t i}\right\rangle, \quad\left\langle J^{i} T^{t j}\right\rangle=\frac{\partial}{\partial h_{t j}}\left\langle J^{i}\right\rangle, \quad\left\langle J^{i} J^{j}\right\rangle=\frac{\partial}{\partial a_{j}}\left\langle J^{i}\right\rangle .
$$

Using the scaling limit $(\omega, k) \rightarrow\left(z^{2} \omega, z k\right)$ for $z \ll 1$ as appropriate for isolating the diffusion pole in the shear channel, the frequency and momentum dependence of the conductivities read

$$
\begin{aligned}
\sigma_{\varepsilon}^{\mathscr{V}}(\omega, k) & =\sigma_{\varepsilon}^{\mathscr{V}} \frac{-D^{2} k^{4}}{\left(\omega+i D k^{2}\right)^{2}} \\
\sigma^{\mathscr{V}}(\omega, k) & =\left(\sigma^{\mathscr{V}}-\frac{\rho \sigma_{\varepsilon}^{\mathscr{V}}}{\varepsilon+P} \frac{\omega}{\omega+i D k^{2}}\right) \frac{i D k^{2}}{\omega+i D k^{2}} \\
\sigma^{\mathscr{B}}(\omega, k) & =\sigma^{\mathscr{B}}-\frac{\rho}{\varepsilon+P}\left[2 \sigma^{\mathscr{V}}-\frac{\rho \sigma_{\varepsilon}^{\mathscr{V}}}{\varepsilon+P} \frac{\omega}{\omega+i D k^{2}}\right] \frac{\omega}{\omega+i D k^{2}}
\end{aligned}
$$

\footnotetext{
${ }^{2}$ For simplicity we consider a fluid with an anomalous charge $U(1)$. The extension to $U_{V}(1) \times U_{5}(1)$ is straightforward.
} 

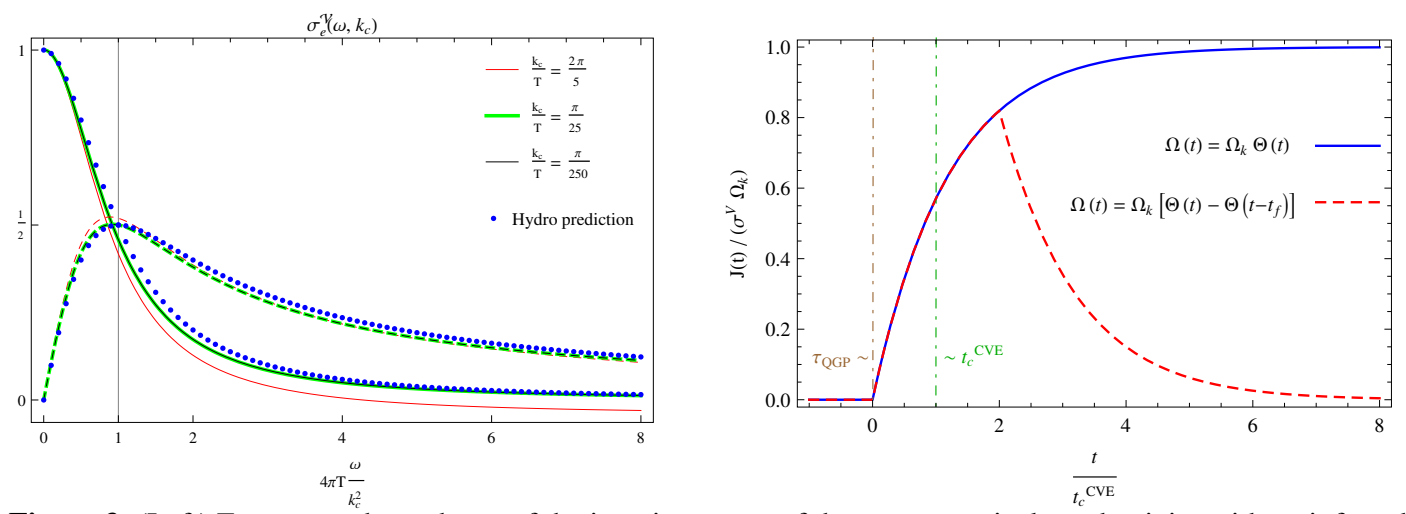

Figure 3. (Left) Frequency dependence of the imaginary part of the vector vortical conductivity with an infrared momentum cut-off. We consider $\tau=95$ and $\mu_{5} / \mu=0.008$. We show as continuous lines the strong coupling results of Sec. 2.2, and as dots the hydrodynamic computation of Sec. 3, cf. Eqs. (14)-(16). The vertical line is the position of the maximum predicted by hydrodynamics. (Right) Induced electric current as a function of time for the CVE as computed in Sec. 4. We consider the time-dependent vorticities $\Omega(t)=\Omega_{k} \Theta(t)$ (continuous blue line), and $\Omega(t)=\Omega_{k}\left(\Theta(t)-\Theta\left(t-t_{f}\right)\right)$ with $t_{f}=2 t_{c}^{\mathrm{CVE}}$ (dashed red line). We consider in Eq. (19) that $\lim _{T \rightarrow \infty}\left(\frac{\rho \sigma_{\varepsilon}^{\mathcal{V}}}{(\varepsilon+P) \sigma_{e}^{\mathscr{V}}}\right)=1 / 6$. The vertical lines are the values of $\tau_{\mathrm{QGP}}$ (brown) and $t_{c}^{\mathrm{CVE}}$ (green). $\tau_{\mathrm{QGP}}$ is indistinguishable from $t=0$ in the scale of the figure.

where the shear diffusion constant is $D=\eta /(\varepsilon+P) \simeq 1 /(4 \pi T)$. The imaginary parts of these conductivities have peaks at $\omega \approx \pm c D k^{2}$, where $c$ is a constant of order 1 . In addition, as a consequence of the coupling to the energy-momentum tensor, the limits $\omega \rightarrow 0$ and $k \rightarrow 0$ do not commute. ${ }^{3}$ We show in Fig. 3 (left) the comparison of these results with the holographic computation of Sec. 2.2. The agreement is remarkable, specially for the conductivities computed with the correlators involving at least one charge current, i.e. $\langle J J\rangle,\langle J T\rangle$ and $\langle T J\rangle$.

\section{Implications for heavy ion physics}

The CVE is the process in which an electric current is induced due to a vortex in the fluid. If one assumes a homogeneous but non-constant vortex, the induced current reads in momentum space

$$
\vec{J}(\omega)=\sigma^{\mathscr{V}}(\omega) \vec{\Omega}(\omega),
$$

where $\vec{\Omega}$ is the vorticity vector. The physical situation in heavy ion collisions does not correspond to rotation driven by an external force, so that to compute $\vec{\Omega}(t)$ (and $\vec{\Omega}(\omega)$ ) one has to solve the hydrodynamic evolution equations, and this is a very difficult problem. Instead, as a first approximation we consider the following estimate

$$
\Omega(t)=\Omega_{k} \Theta(t) \Longrightarrow \Omega(\omega)=\Omega_{k} \frac{i}{2 \pi} \frac{1}{\omega+i \varepsilon},
$$

where $\Theta$ is the step function. Then after plugging Eqs. (15) and (18) into Eq. (17) and performing the inverse Fourier transform, the induced electric current is

$$
J(t)=\Theta(t) \Omega_{k}\left[\sigma^{\mathscr{V}}\left(1-e^{-D k^{2} t}\right)-\frac{\rho \sigma_{\varepsilon}^{\mathscr{V}}}{\varepsilon+P} D k^{2} t e^{-D k^{2} t}\right] .
$$

\footnotetext{
${ }^{3}$ In [19] it has been found the commutativity of these limits in $\sigma^{\mathscr{B}}(\omega, k)$ within a weakly coupled approach. However, this computation does not take into account the coupling to the energy-momentum tensor.
} 
This formula is characterized by a decay/production time $t_{c}^{\mathrm{CVE}} \simeq 1 / D k^{2} \simeq 4 \pi T L^{2}$ (see Fig. 3 (right)). In heavy ion collisions at RHIC and LHC the typical values of temperature and size of the fireball are $T \simeq 350 \mathrm{MeV}$ and $L \simeq 10 \mathrm{fm}$ respectively. This means that

$$
\tau_{\mathrm{QGP}} \simeq 10 \mathrm{fm} / \mathrm{c} \ll t_{c}^{\mathrm{CVE}} \simeq 2200 \mathrm{fm} / \mathrm{c},
$$

where $\tau_{\mathrm{QGP}}$ is the typical lifetime of the quark-gluon plasma (QGP). As a consequence, there is essentially no response of the system during the time scale of the QGP, and one would expect the CVE being negligible in the QGP as created in heavy ion collisions. A definitive answer would demand, however, to study the CVE dynamically as mentioned above.

The induced electric current in a magnetic field, i.e. the CME, was studied in [1]. In this case it is safer to consider the magnetic field as an external force. An estimate similar to the one performed in this section leads to a much faster response of $J(t)$, such that $t_{c}^{\mathrm{CME}} \lesssim \tau_{\mathrm{QGP}}$. Then one can conclude that the CME is effectively produced within the lifetime of the QGP, in agreement with [1].

\section{Conclusions}

Using the Kubo formulae formalism, we have studied the anomalous transport properties of a relativistic fluid in presence of external magnetic fields and vortices. The CVC shows either at weak and strong coupling a frequency dependence in the form of a delta centered at zero frequency. Using hydrodynamic considerations we have identified also the presence of a shear diffusion pole in the CMC and CVC. Finally, contrary to the CME, a first estimate indicates that the CVE could be negligible in heavy ion collisions which, however, requires confirmation via numerical methods. We hope that the present work will stimulate further developments in the field.

\section{References}

[1] D.E. Kharzeev, H.J. Warringa, Phys. Rev. D80, 034028 (2009).

[2] D.T. Son, P. Surowka, Phys. Rev. Lett. 103, 191601 (2009).

[3] B. Keren-Zur, Y. Oz, JHEP 06, 006 (2010).

[4] G. Basar, D.E. Kharzeev, H.U. Yee, Phys. Rev. B89, 035142 (2014).

[5] K. Landsteiner, E. Megias, F. Pena-Benitez, Phys. Rev. Lett. 107, 021601 (2011).

[6] K. Landsteiner, E. Megias, L. Melgar, F. Pena-Benitez, JHEP 1109, 121 (2011).

[7] S. Chapman, Y. Neiman, Y. Oz, JHEP 1207, 128 (2012).

[8] E. Megias, F. Pena-Benitez, JHEP 1305, 115 (2013).

[9] K. Jensen, R. Loganayagam, A. Yarom, JHEP 1302, 088 (2013).

[10] J. Erdmenger, M. Haack, M. Kaminski, A. Yarom, JHEP 01, 055 (2009).

[11] N. Banerjee et al., JHEP 01, 094 (2011).

[12] S. Bhattacharyya, J.R. David, S. Thakur, JHEP 1401, 010 (2014).

[13] E. Megias, M. Valle (2014), 1408.0165.

[14] I. Amado, K. Landsteiner, F. Pena-Benitez, JHEP 1105, 081 (2011).

[15] K. Landsteiner, E. Megias, F. Pena-Benitez, Lect. Notes Phys. 871, 433 (2013).

[16] J.L. Manes, M. Valle, JHEP 1301, 008 (2013).

[17] K. Landsteiner, E. Megias, F. Pena-Benitez (2013), 1312 . 1204.

[18] D.T. Son, A.O. Starinets, JHEP 0209, 042 (2002).

[19] D. Satow, H.U. Yee, Phys. Rev. D90, 014027 (2014). 